\title{
A Simplified Method for the Estimation of Hole Free Volume Fraction from the Specific Volume
}

\author{
G. DlubeK ${ }^{a}$ And J. Pionteck ${ }^{b}$ \\ ${ }^{a}$ ITA Institut für Innovative Technologien, Köthen, Aussenstelle Halle \\ Wiesenring 4, D-06120 Lieskau (bei Halle/S.), Germany \\ ${ }^{b}$ Leibniz-Institut für Polymerforschung Dresden e.V. \\ Hohe Strasse 6, D-01069 Dresden, Germany

\begin{abstract}
A brief introduction in the equation of state of the Simha-Somcynsky hole theory is presented. This theory allows to calculate the volume fraction $h$ of free volume holes from pressure-volume-temperature experiments. These holes are detected by ortho-positronium and from the value of $h$ and the mean ortho-positronium hole size the hole density may be calculated. We discuss a simplified method to estimate $h$ which makes use of the relation $V^{*}=1.52 V_{\mathrm{W}}$ between the scaling volume $V^{*}$ of the Simha-Somcynsky hole theory equation of state and the van der Waals volume $V_{\mathrm{W}}$. Moreover, we present a new simplified method for the estimation of $h$ which starts with the Schottky (Arrhenius) equation for the concentration of thermal vacancies and makes use of a linear relation between the scaling temperature $T^{*}$ and the hole formation enthalpy $H_{\mathrm{h}}, H_{\mathrm{h}}(\mathrm{kJ} / \mathrm{mol})=6.54 \times 10^{-4} T^{*}(\mathrm{~K})$.
\end{abstract}

PACS numbers: 61.41.+e, 78.70.--g

\section{Introduction}

Positron annihilation lifetime spectroscopy (PALS) is the most important experimental method to measure the mean volume of subnanometre holes which form in amorphous polymers the hole (or excess) free volume and, with larger limitations, their size distribution [1]. The method, however, cannot deliver directly the hole density and the fraction of holes. A method which proved to be very successful in estimation of the fraction of free volume holes is the analysis of pressurevolume-temperature (PVT) experiments using the Simha-Somcynsky hole theory equation of state (S-S eos) [2-4]. This theory describes the structure of a liquid by a cell or lattice model (hexagonal dense packed, hdp) which allows an occupied lattice-site fraction $y$ of less than one. The hole fraction is given by $h=1-y$. 
The value of $y$ is obtained through the pressure equation $P=-(\partial F / \partial V)_{T}$ and the minimisation condition $(\partial F / \partial y)_{V, T}=0$. The S-S theory has been successfully applied to analyse the volumetric behaviour of low molecular liquids, linear and non-linear polymers, and also of copolymers and blends. It was found that the vacancies of the $\mathrm{S}-\mathrm{S}$ lattice form multivacancies or agglomerates due to their large concentration [5]. Ortho-positronium (o-Ps) annihilates from a state localised at one of theses multivacancies [6-14]. From its mean size, $\left\langle\nu_{\mathrm{h}}\right\rangle$, and the hole fraction $h$ a realistic value for the hole density $N_{\mathrm{h}}=h /\left\langle\nu_{\mathrm{h}}\right\rangle$ can be estimated. In this work we focus our attention to approximations, which allow the estimation of $h$ and its temperature dependence from a single density measurement.

\section{A simplified method to estimate the hole free volume fraction} form

The pressure equation $P=-(\partial F / \partial V)_{T}$ of the $\mathrm{S}-\mathrm{S}$ eos has for $P \rightarrow 0$ the

$$
\tilde{T}=2 y(y \tilde{V})^{-2}\left[1.2045-1.1011(y \tilde{V})^{-2}\right]\left[1-2^{-1 / 6} y(y \tilde{V})^{-1 / 3}\right] .
$$

$\tilde{V}$ and $\tilde{T}$ are reduced variables, $\tilde{V}=V / V^{*}, \tilde{T}=T / T^{*}$, where $V^{*}$ and $T^{*}$ are scaling parameters characteristic of the material. It was shown that both S-S eos equations, $P=-(\partial F / \partial V)_{T}$ and $(\partial F / \partial y)_{V, T}=0$, may be replaced by the universal interpolation expression

$$
\ln \tilde{V}=a+b \tilde{T}^{3 / 2},
$$

where for polymers $a=-0.10346$ and $b=23.854$ [3]. From a linear fit to the plot of $\ln V$ vs. $T^{3 / 2}, \ln V=A+B T^{3 / 2}$, the scaling parameters $V^{*}=\exp (A+0.10346)$ and $T^{*}=(23.854 / B)^{2 / 3}$ can be determined. With the known $V^{*}$ and $T^{*}$ the hole fraction $h$ can be calculated numerically from Eq. (1) for a given $V$ and $T$.

Equation (1) is derived under the general assumption of equilibrium, however, the specific assumption that the free energy is a minimum, has not been made. Therefore, it is usual to calculate the $h=1-y$ values from the specific volume also below $T_{\mathrm{g}}$ via Eq. (1) using the scaling parameters determined from the experiments above $T_{\mathrm{g}}$. These $h$ values are considered to be sufficiently good approximations for conditions not too far from equilibrium.

It was shown that in equilibrium, $T>T_{\mathrm{g}}$, the hole fraction $h$ can be calculated from the approximation [2-4]:

$$
h=1-V_{\text {occ }} / V \text {, where } V_{\text {occ }}=K V^{*} .
$$

The $\mathrm{S}-\mathrm{S}$ eos shows that $K$ is a material independent, very slowly varying function of $T$ which can be approximated by its mean value of $K=0.956$. The almost constancy of $K$ implies that $V_{\text {occ }}$ shows practically no thermal expansion. This is only true above $T_{\mathrm{g}}$. For $T<T_{\mathrm{g}}, V_{\text {occ }}$ varies approximately with half of the total thermal expansion coefficient of the glass $[9,11]$.

For an approximate estimation of the material constant $V^{*}$ we use a method described by Simha and Carri [4]. These authors observed a linear relation between the van der Waals volume $V_{\mathrm{W}}$ and $V^{*}$ and analysed $V^{*}=1.45 V_{\mathrm{W}}$ for polymers 
and $V^{*}=1.60 V_{\mathrm{W}}$ for low molecular liquids. The $V^{*}$-values estimated by other groups [6-8] for several polymers correspond to $V^{*} / V_{\mathrm{W}}=1.57-1.60$.

In Fig. 1 we have plotted $V^{*}$ taken from our previous studies [9-14] vs. $V_{\mathrm{W}}$, which was calculated from the group contributions listed by van Krevelen [15]. A linear fit to all data shown in the figure delivers $V^{*}=-0.0065( \pm 0.027)+$ $1.537( \pm 0.05) V_{\mathrm{W}}$. A fit constrained to pass zero gives

$$
V^{*}=1.520( \pm 0.013) V_{\mathrm{W}} .
$$

The maximum deviation from this line is shown by PDMS (1.68) and monomeric and oligomeric DGEBA (1.44). From our data the relation

$$
V_{\text {occ }}=1.453 V_{\mathrm{W}}
$$

follows. It is noteworthy that this value agrees with the typical volume of polymer crystals estimated by van Krevelen to be $V_{\mathrm{c}}(298 \mathrm{~K}) \approx 1.45 V_{\mathrm{W}}[15]$. It is distinctly larger than the occupied volume estimated traditionally from the extrapolation of the specific volume of liquid (rubbery, Doolittle, see [15]) or crystalline (Bondi, see [15]) materials down to $0 \mathrm{~K}, V_{\mathrm{occ}}(0)=V_{\mathrm{r}}(0) \approx V_{\mathrm{c}}(0) \approx 1.3 V_{\mathrm{W}}$.

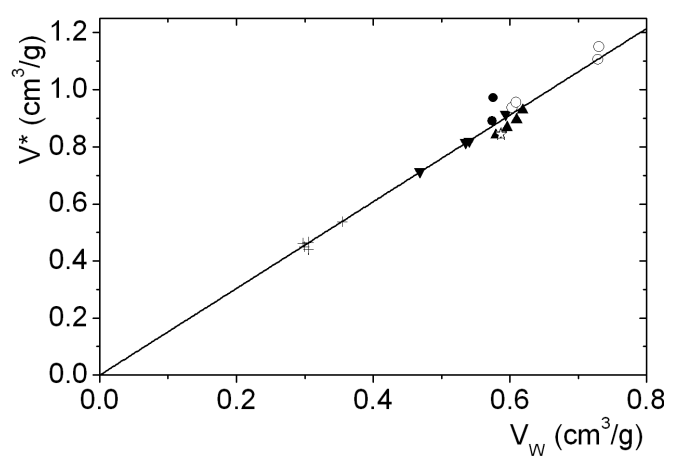

Fig. 1. The relation between the S-S eos scaling volume $V^{*}$ and the van der Waals volume $V_{\mathrm{W}}$ for polymers. Shown are the data for hydrogen polymers (PIB, aPP, PS, COC, open circles [9]), siloxanes (PDMS, PMPS, filled circles [10]), polyalkylmethacrylates (PMMA, PEMA, PPMA, PBMA, filled up triangles [11]), monomeric and oligomeric DGEBA (open stars [12]), fluoro polymers (VDF/HFP $22, \mathrm{PFE}, \mathrm{CY}$ TOP, Teflon AF16000 and AF2400, crosses [13]), and further polymers (PC, SAN35, SMA50, PVC [9, 14], filled down triangles; for the nomenclature of polymers see our previous papers and van Krevelen [15]). The line is a linear fit to the data constrained to pass zero.

We remark here that Eqs. (1)-(5) lead to the relation between the hole fraction $h=1-V_{\mathrm{occ}} / V=1-1.453 V_{\mathrm{W}} / V$ and the fraction of the total (van der Waals) free volume $f_{\mathrm{W}}=1-V_{\mathrm{W}} / V$ :

$$
h=1.453 f_{\mathrm{W}}-0.453 \text {. }
$$

For $h=0$ the interstitial free volume $f_{\mathrm{i}}=f_{\mathrm{W}}=0.318$ follows. In order to estimate 
the temperature dependence of $h$ on Eq. (3) we have to know the variation of $V$ with $T$. We can obtain this from Eq. (2) for $T>T_{\mathrm{g}}$. With the known $V^{*}$ one may estimate $T^{*}$ from the value of $V$ at a given $T$ via

$$
T^{*}=T\left[b /\left(\ln V / V^{*}-a\right)\right]^{2 / 3} .
$$

With the known values of $V^{*}$ and $T^{*}$ we may estimate $h(T)$ either from Eq. (1) or from the approximation Eq. (3) calculating the total volume $V(T)$ from Eq. (2).

Another way, the relation is used

$$
h(T)=a_{0}+a_{1} \tilde{T}+a_{2} \tilde{T}^{2}
$$

derived in the paper of Utracki and Simha [3] where $a_{0}=-0.09211, a_{1}=4.892$, and $a_{2}=12.56$. Again, Eq. (8) is only valid for the equilibrium $T>T_{\mathrm{g}}$. Equation (1) can be applied also for the temperature range $T<T_{\mathrm{g}}$, but for the calculation the temperature dependence of $V$ must be known.

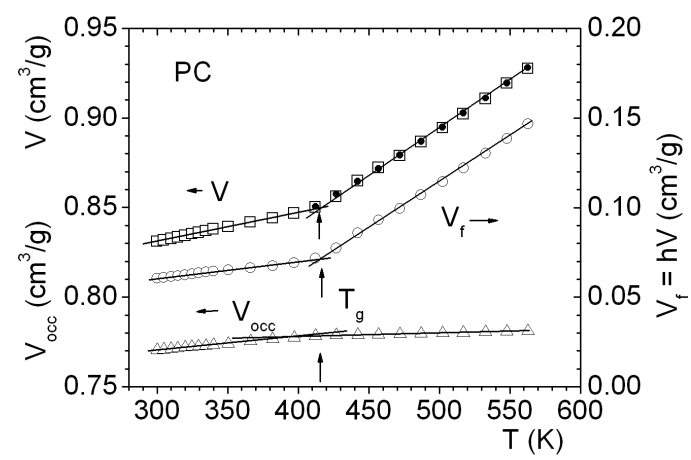

Fig. 2. The specific total, $V$, free, $V_{\mathrm{f}}=h V$, and occupied, $V_{\text {occ }}=(1-h) V$, volume of polycarbonate (PC) as a function of the temperature $T$ at ambient pressure. Open symbols: specific volumes, dots: Simha-Somcynsky equation of state fits to the total volume in the temperature range $T>T_{\mathrm{g}}$. The corresponding values of $h$ are shown in Fig. 3 as open symbols.

As an example we show in Figs. 2 and 3 results from PVT data of PC measured by us with a Gnomix mercury dilatometer (for experimental details see [9-14]). The fit of the experimental PVT data by Eq. (2) in the temperature range $T>T_{\mathrm{g}}$ delivers the scaling parameters $V^{*}=0.8146( \pm 0.003) \mathrm{cm}^{3} / \mathrm{g}$ and $T^{*}=12300( \pm 50) \mathrm{K}$. Figure 2 shows the total, $V$, the occupied, $V_{\text {occ }}=y V=$ $(1-h) V$, and the hole free, $V_{\mathrm{fh}}=V_{\mathrm{f}}=h V$, volumes. The hole fraction $h$ was calculated from Eq. (1). All of volume data show clearly the change in their increments at the glass transition $T_{\mathrm{g}}=415 \mathrm{~K}$. We observe that the coefficient of thermal expansion of the occupied volume corresponds to $\alpha_{\text {occ, } \mathrm{g}} \approx \alpha_{\mathrm{g}} / 2=$ $1 \times 10^{-4} \mathrm{~K}^{-1}$ in the glass $(\mathrm{g})$ and $\alpha_{\text {occ }, \mathrm{r}}=0.2 \times 10^{-4} \mathrm{~K}^{-1} \approx 0$ in the rubber (r). This is unexpected in the light of classical free volume models, but was also found recently for other polymers $[9,11-13]$. This result has a particular 


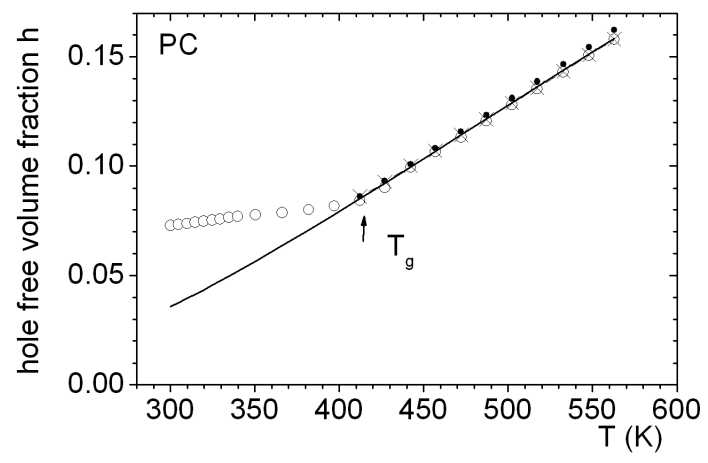

Fig. 3. Fractional hole free volume, $h=V_{\mathrm{f}} / V$, for PC calculated from PVT data employing the Simha-Somcynsky theory. Open symbols: from the equation of state Eq. (1), dots: from the approximation Eq. (3), crosses: from the approximation Eq. (8), line: from approximation Eq. (9).

importance since it shows that the usual expression for the fractional coefficient of the free volume expansion in the rubbery state, $\alpha_{\mathrm{fr}}^{*} \approx \Delta \alpha=\alpha_{\mathrm{r}}-\alpha_{\mathrm{g}}$, is not a good approximation but should be rather substituted by $\alpha_{\mathrm{fr}}^{*} \approx \alpha_{\mathrm{r}}$. The fractional coefficient $\alpha_{\mathrm{f}}^{*}$ is defined by $\alpha_{\mathrm{f}}^{*}=(1 / V)\left(\mathrm{d} V_{\mathrm{f}} / \mathrm{d} T\right)_{P}=h \alpha_{\mathrm{f}}\left(\alpha=\alpha_{\text {occ }}^{*}+\alpha_{\mathrm{f}}^{*}\right.$ and $\left.\alpha_{\text {occ }}^{*}=(1-h) \alpha_{\text {occ }}\right)$. With the scaling parameters $V^{*}$ and $T^{*}$ we have calculated the hole fraction in the approximation of Eq. (3) and Eq. (8) for the temperature range $T>T_{\mathrm{g}}$. These data are shown in Fig. 3 as dots and crosses. It can be observed that both approximations agree well with the $h$-values calculated from Eq. (1), where Eq. (8) gives a better approximation than Eq. (3). Since the ratio $V^{*} / V_{\mathrm{W}}=0.8146 / 0.535=1.523$ does not differ from Eq. (4), the values calculated from Eqs. (3)-(8) do not differ much from the exact solution of Eqs. (1) and (2).

This may be, however, not always the case. For PS, for example, we determined from Eq. (2) $V^{*}=0.9373( \pm 0.002) \mathrm{cm}^{3} / \mathrm{g}$ and $T^{*}=11650( \pm 40) \mathrm{K}$. The $V^{*}$ corresponds to $V^{*} / V_{\mathrm{W}}=0.9373 / 0.6033=1.554$. From Eq. (4) $V^{*}=0.9170 \mathrm{~cm}^{3} / \mathrm{g}$ would follow, which gives with $V=0.9660 \mathrm{~cm}^{3} / \mathrm{g}$ at $T_{\mathrm{g}}=371 \mathrm{~K}$ from Eq. (7) a scaling temperature of $T^{*}=12288 \mathrm{~K}$. With these parameters $h\left(T_{\mathrm{g}}\right)=0.093$ follows while the exact solution from Eqs. (1) and (2) is $h\left(T_{\mathrm{g}}\right)=0.073$. An underestimation of $V^{*}$ by $1 \%$ would lead to an underestimation of the occupied volume fraction $y=V_{\text {occ }} / V$ by $1.7 \%$ but to an overestimation of the hole volume fraction $h$ by $15 \%$ since $\mathrm{h}$ is calculated from the difference $h=1-y \approx 0.1$.

\section{Another simplified method to estimate the hole free volume fraction}

The holes of the S-S lattice may be considered like thermal vacancies (the Schottky defects) in a monoatomic crystal of hdp structure. Then we may expect that the hole fraction (or the atomic vacancy concentration) $h$ follows the Schottky (Arrhenius) equation $h=A \exp \left(-H_{\mathrm{h}} / R T\right)$ where $H_{\mathrm{h}}$ is the hole formation enthalpy per mole $\mathrm{S}-\mathrm{S}$ mer, $A$ is a pre-exponential factor, and $R$ is the 
gas constant. In our recent works $[9,10,13]$ we have shown that this approach works well and that $H_{\mathrm{h}}=(0.35-0.55) E_{\mathrm{c}}$ where Ec is the cohesive energy of a S-S mer. The S-S theory defines the scaling temperature $T^{*}$ to be linearly related to the attraction energy between a pair of $\mathrm{S}-\mathrm{S}$ mers. We may therefore expect that $H_{\mathrm{h}}$ is linearly related to $T^{*}$. In Fig. 4 we have plotted the values of $H_{\mathrm{h}}$ determined in our previous works $[9-14]$ vs. $T^{*}$. A linear fit to the data delivers $H_{\mathrm{h}}=-0.02( \pm 0.4)+6.56( \pm 0.4) \times 10^{-4} \mathrm{~T}^{*}$. When constraining the fit to pass zero we obtain $H_{\mathrm{h}}=6.54( \pm 0.06) \times 10^{-4} \mathrm{~T}^{*}$. Here $H_{\mathrm{h}}$ is given in $\mathrm{kJ} / \mathrm{mol}$ and $T^{*}$ in $\mathrm{K}$.

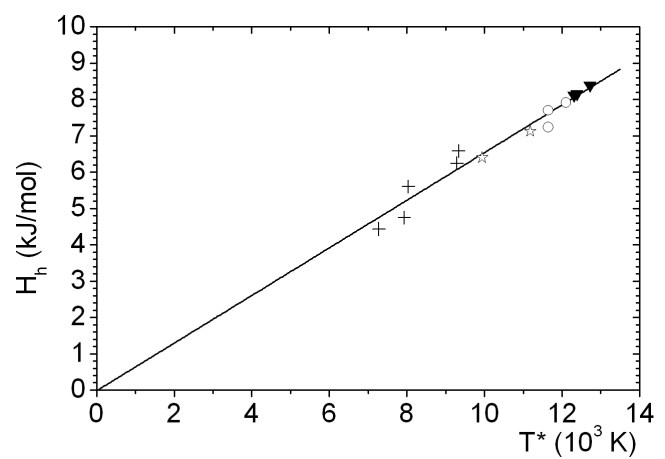

Fig. 4. Hole formation enthalpy $H_{\mathrm{h}}$ estimated from the analysis of PVT experiments with the $\mathrm{S}-\mathrm{S}$ eos as a function of the scaling temperature $T^{*}$. Symbols as in Fig. 1.

For a further estimation of $H_{\mathrm{h}}$ we express the hole fraction by the equation

$$
h=A \exp (-B / \tilde{T}),
$$

where the hole formation enthalpy $H_{\mathrm{h}}$ is given by $H_{\mathrm{h}}=\mathrm{BT}^{*} \mathrm{R}$ and $\tilde{T}=T / T^{*}$ is the reduced temperature. We may obtain a general value of $B$ by fitting this function to the numerical solution $h=h(\tilde{T})$ of Eqs. (1) and (2). A fit in the temperature range $1.6<100 \tilde{T}<6.74$ under constraining $A=1$ delivers $B=$ $8.513( \pm 0.07) \times 10^{-2}$ (corresponding to $\left.H_{\mathrm{h}}=7.08 \times 10^{-4} \mathrm{~T}^{*}\right)$. Figure 5 shows that this fit describes the general behaviour of $h$ as a function of $T$ but the fit is not very perfect $\left(r^{2}=0.9834\right)$. An improvement may be obtained by allowing a temperature dependence of $B$ (which is related to the temperature dependence of the cohesive energy $E_{\mathrm{c}}$ ) or by constraining the range of fit. For the fitting range $2.1<100 \tilde{T}<5.0$ (corresponding to $0.02<h<0.18$ ) and allowing a variation also in $A$ we obtained $A=0.869( \pm 0.01)$ and $B=7.786( \pm 0.03) \times 10^{-2}$. The fit is shown in Fig. 5 as dots. The fit is very sufficient $\left(r^{2}=0.9997\right)$ and the value of $B$ corresponds to $H_{\mathrm{h}}=6.47 \times 10^{-4} T^{*}$ which is in agreement with the fit in Fig. 4 .

Figure 3 includes the data from the approximation Eq. (9) as a solid line. Here we have extrapolated $h$ to temperatures below $T_{\mathrm{g}}$ to show the expected behaviour of $h$ in quasi-equilibrium. The difference of this line and the experiment (open symbols) shows the fraction of frozen-in holes. The discussed approximations open the way to calculate the scaling parameters $V^{*}$ and $T^{*}$ from a single 


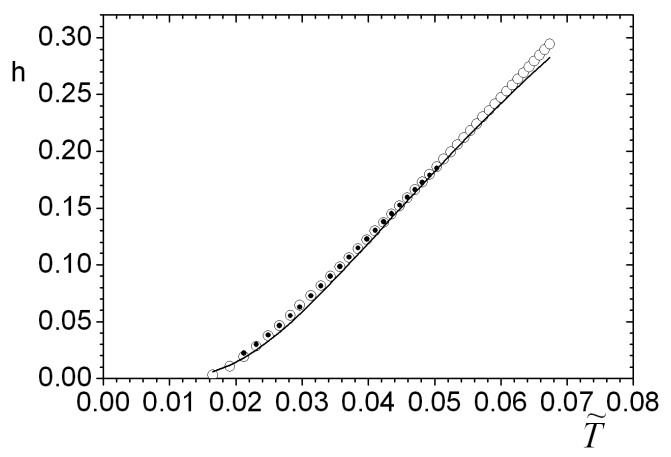

Fig. 5. Fit of Eq. (9), $h=A \exp (-B / \tilde{T})$, to the hole fraction $h$ from numerical solution of the S-S eos Eqs. (1) and (2) (open circles). $\tilde{T}=T / T^{*}$ is the reduced temperature. Solid line: fitting range $1.6<100 \tilde{T}<6.74, A=1$ constrained, $B=8.513( \pm 0.7) \times 10^{-2}$. Dots: fitting range $2.1<100 \tilde{T}<5.0, A=0.869( \pm 0.01), B=7.786( \pm 0.3) \times 10^{-2}$.

density measurement and to determine the temperature dependence of the hole free volume fraction $h$.

\section{Acknowledgments}

The authors thank R. Krause-Rehberg, E.M. Hassan, M.Q. Shaikh, S. Thränert, V. Bondarenko (Univ. of Halle), M.A. Alam, S. Townrow, D. Kilburn, D. Bamford (Univ. of Bristol), T. Goworek, J. Wawryszczuk, M. Sniegocka (Univ. of Lublin), and F. Faupel , K. Rätzke, J. Kruse (Univ. of Kiel) for the fruitful and continuous collaboration in the field of PALS.

\section{References}

[1] Principles and Application of Positron and Positronium Chemistry, Eds. Y.C. Jean, P.E. Mallon, D.M. Schrader, World Scientific, Singapore 2003.

[2] R. Simha, T. Somcynsky, Macromolecules 2, 342 (1969).

[3] L.A. Utracki, R. Simha, Macromol. Theory Simul. 10, 17 (2001).

[4] R. Simha, G. Carri, J. Polym. Sci., Part B: Polym. Phys. 32, 2645 (2094).

[5] S. Vleeshouwers, J.-E. Kluin, J.D. McGervey, A.M. Jamieson, R. Simha, J. Polym. Sci., Part B: Polym. Phys. 30, 1429 (1992).

[6] R. Srithawatpong, Z.L. Peng, B.G. Olson, A.M. Jamieson, R. Simha, J.D. McGervey, T.R. Maier, A.F. Halasa, H. Ishida, J. Polym. Sci., Part B: Polym. Phys. 37, 2754 (1999).

[7] M. Schmidt, F.H.J. Maurer, Macromolecules 33, 3879 (2000).

[8] G. Consolati, F. Quasso, R. Simha, B. G. Olson, J. Polym. Sci., Part B: Polym. Phys. 43, 2225 (2005). 
[9] G. Dlubek, J. Pionteck, D. Kilburn, Macromol. Chem. Phys. 205, 500 (2004); G. Dlubek, V. Bondarenko, I.Y. Al-Qaradawi, D. Kilburn, R. Krause-Rehberg, Macromol. Chem. Phys. 205, 512 (2004); D. Kilburn, J. Wawryszczuk, G. Dlubek, J. Pionteck, R. Häßler, M.A. Alam, Macromol. Chem. Phys. 207, 721 (2006).

[10] G. Dlubek, U. De, J. Pionteck, N.Yu. Arutyunov, M. Edelmann, R. KrauseRehberg, Macromol. Chem. Phys. 206, 827 (2005); G. Dlubek, M.Q. Shaikh, R. Krause-Rehberg, M. Paluch, J. Chem. Phys. 126, 024906 (2007).

[11] D. Kilburn, G. Dlubek, J. Pionteck, M.A. Alam, Polymer 47, 7774 (2006).

[12] G. Dlubek, E.M. Hassan, R. Krause-Rehberg, J. Pionteck, Phys. Rev. E 75, 031803 (2006); G. Dlubek, J. Pionteck, M.Q. Shaikh, E.M. Hassan, R. KrauseRehberg, Phys. Rev. E 75, 021802 (2006).

[13] M. Rudel, J. Kruse, K. Raetzke, F. Faupel, Yu.P. Yampolskii, V.P. Shantarovich, G. Dlubek, Macromolecules 41, 788 (2008); G. Dlubek, J. Pionteck, J. Kruse, K. Rätzke, F. Faupel, Y. Yampolskii, V.P. Shantarovich, submitted to Macromolecules.

[14] D. Kilburn, G. Dlubek, J. Pionteck, D. Bamford, M.A. Alam, Polymer 46, 859, 869 (2005).

[15] D.W. van Krevelen, Properties of Polymers, Elsevier Sci. Publ. Co., Amsterdam 1990. 